\title{
Alinhamento entre estrutura organizacional de projetos e estratégia de manufatura: uma análise comparativa de múltiplos casos
}

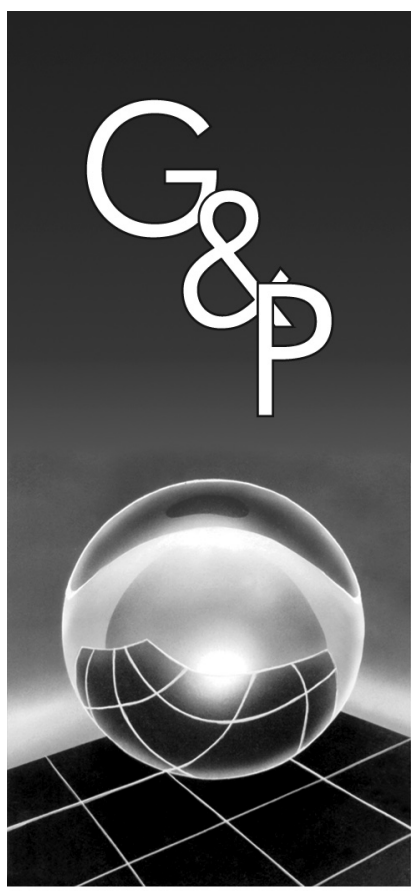

Leandro Alves Patah

Marly Monteiro de Carvalho
Resumo

O principal objetivo deste trabalho é estabelecer uma relação entre a estrutura de gerenciamento de projetos de uma organização e sua estratégia de manufatura. Pretende-se verificar também se existe um alinhamento entre a configuração da estrutura organizacional e o tipo de estratégia de manufatura de uma organização. A abordagem metodológica utilizada foi o estudo de múltiplos casos, realizado em três empresas dos setores de cosméticos, eletro-eletrônico e aeroespacial. Os principais resultados apontam para estruturas mais complexas de gerenciamento de projetos à medida que se caminha no continuum entre volume e variedade.

Palavras-chave: Gerenciamento de projetos. Estratégia de manufatura. Estrutura organizacional. Escritórios de projetos.

\section{Introdução}

Cada vez mais, novos bens e serviços são produzidos por meio de projetos implementados pelas empresas, evidenciando o uso, cada vez mais amplo, de metodologias de gerenciamento de projetos. Além disso, King (1993) argumenta que os projetos permitem alinhar as estratégias e desdobrá-las para todas as áreas da corporação.

Com isso, é necessária uma estratégia gerencial que utilize as unidades operacionais para a execução do trabalho e a medição do desempenho que analise a eficiência do trabalho que está sendo realizado e gere informações para os gerentes seniores. A gerência de projetos faz tudo isso e, portanto, é o caminho escolhido para gerenciar os aspectos críticos dos negócios (CLELAND; IRELAND, 2000).

Além disso, Platts e Mills (2002) argumentam que um processo de sucesso de criação de estratégias é mais que uma sequência de passos, ele precisa de outras características, como um bom gerenciamento de projetos. Por outro lado, na literatura de estratégia, já existe um consenso das necessidades de alinhamento entre estratégia e estrutura (MINTZBERG; HEYDEN, 1999; MINTZBER; GWATERS, 1985).

Nesse sentido, diversos autores têm se empenhado em estudar a importância da estrutura de gestão de projetos para seu sucesso (LARSON; GOBELI, 1999; KELLER, 2001, CARDINAL; MARLE, 2006).
No contexto de projetos, Galbraith (1971) distinguiu diferentes tipos de estrutura, em um contínuo no qual em um extremo está a organização funcional e no outro extremo encontra-se a organização por projeto. Diversos autores utilizam a classificação em três tipos de estruturas básicas que aplicam os conceitos de gerenciamento de projetos em uma organização: a estrutura funcional, a estrutura matricial e a estrutura projetizada (MEREDITH; MANTEL, 2000; KERZNER, 2001; PATAH; CARVALHO, 2002; CARVALHO; RABECHINI Jr., 2005). A escolha entre esses tipos de estrutura exigem cautela, pois estudos apontam que o tipo de estrutura afeta o resultado do projeto, dado que a organização funcional apresenta menos sucesso do que a matricial e a projetizada (LARSON; GOBELI, 1999).

No entanto, uma preocupação recente da literatura de gestão de projetos é a tendência de abordar projetos a partir de uma perspectiva de projetos únicos, tratando a unidade de análise como um fenômeno isolado, uma ilha (ENGWALL, 2003). Desta forma, o contexto organizacional que envolve o projeto e as estratégias organizacionais costuma ficar fora do contexto de análise.

Uma estrutura que pretende fazer uma ponte entre o projeto e a organização como um todo é o escritório de gestão de projetos (Project Management Office - PMO). 
O PMO consiste em uma estrutura voltada para a aplicação dos conceitos de gerenciamento de projetos dentro de uma organização, podendo assumir diferentes funções junto a ela: desde um simples setor para o auxílio no controle de projetos, até um departamento da empresa pelo qual passam todos os projetos gerenciados pela organização. Segundo DAI (2001), o PMO pode ser definido com uma entidade organizacional estabelecida para auxiliar os gerentes de projeto e os times da organização na implementação dos princípios, práticas, metodologias, ferramentas e técnicas do gerenciamento de projetos.

Um gerenciamento por projetos bem-sucedido exige o preenchimento da lacuna entre a visão da empresa e os projetos em andamento (DINSMORE, 1998). É neste sentido que o PMO pode auxiliar, sobremaneira, a transformação das estratégias de uma empresa em resultados pelo gerenciamento de projetos. Ele pode auxiliar na obtenção e avaliação das informações, na avaliação e seleção das estratégias e no controle da implementação das estratégias por meio da execução com sucesso dos projetos, resultando no cumprimento das metas da organização.

Cinco modelos de PMO são propostos por Dinsmore (1998): Autonomous Project Team (APT); Project Support Office (PSO); Project Management Center of Excellence (PMCOE); Program Management Office (PrgMO); e o Chief Project Officer (CPO). O modelo APT apresenta-se quando uma organização realiza alguns projetos autônomos e a função de gerenciamento de projetos permanece dentro da própria equipe. O PSO é primariamente uma estrutura de suporte, que fornece apoio técnico e administrativo, ferramentas e serviços aos vários gerentes de projeto da organização. O Project Management Center of Excellence é uma estrutura que centraliza o conhecimento em gerenciamento de projetos em uma organização e cuja responsabilidade consiste em aplicar este conhecimento. O Program Management Office apresenta-se como uma organização que aglutina os gerentes de projetos e é responsável pelos resultados dos projetos. O CPO consiste em um papel desempenhado por um membro da diretoria, responsável por administrar o portfolio de projetos da organização.

O principal objetivo deste trabalho é estabelecer uma relação entre a estrutura de gerenciamento de projetos de uma organização e sua estratégia de manufatura, possibilitando gerar um quadro analítico que associe os modelos de estruturas e a estratégia da empresa, a respeito do papel das atividades de gerenciamento de projetos como fonte de vantagem competitiva e obter elementos de suporte para a seleção da melhor estrutura organizacional. Pretende-se verificar também a configuração da estrutura mais adequada às estratégias de manufatura de uma organização.

A abordagem metodológica utilizada foi o estudo de caso, realizado em três diferentes empresas. Após a condução dos estudos de caso, procurou-se realizar uma análise comparativa entre os três, buscando-se demonstrar a aplicação do modelo de alinhamento da matriz volumevariedade em suas principais regiões.

Este artigo está estruturado em 5 seções. A seção 2 apresenta o quadro teórico que suportou a pesquisa, destacando o contexto organizacional da gestão de projetos, as estruturas básicas e os escritórios de projeto. Na seção 3 são apresentados oframework proposto neste trabalho e os principais aspectos metodológicos da pesquisa de campo. A seção 4 apresenta os resultados dos estudos de caso e a discussão da pesquisa de campo, respectivamente. Finalmente, a seção 5 traz as conclusões e recomendações.

\section{Síntese da discussão teórica: estratégia, estrutura e projetos}

O gerenciamento de projetos foi desenvolvido como um conceito de liderança de atividades intersetoriais com o objetivo de solucionar um problema temporário. Esta característica permite atingir um alto grau de inovação nas soluções apresentadas para tarefas cada vez mais complexas (LITKE, 1995).

Nas mais diversas áreas de aplicação, bens e serviços novos são produzidos por projetos, o que pode ser demonstrado pelo aumento do número de empresas que estão adotando a metodologia de gerenciamento de projetos (KERZNER, 2001).

Segundo o Project Management Institute PMI (2004), um projeto pode ser definido com um esforço temporário para criar um bem ou serviço único e o gerenciamento de projetos pode ser definido como a arte de coordenar atividades com o objetivo de atingir as expectativas dos stakeholders.

Segundo Carvalho e Rabechini Jr. (2005), em todas as definições de projeto podem-se perceber dois conceitos intrínsecos: um referente à temporalidade, ou seja, todo projeto tem um começo e um fim bem determinado; e outro que se refere à unicidade ou singularidade, ou seja, que o bem ou serviço é, de algum modo, diferente de todos os similares feitos anteriormente. Contudo estes autores destacam que, embora não tão explícitas em todas as suas definições, a incerteza e a complexidade inerentes a eles são também questões fundamentais para compreender este conceito e dimensionar o aparato gerencial necessário para atingir o sucesso em projetos. Em trabalhos e pesquisas recentes estes autores argumentam que é necessário fazer uma adequada categorização dos projetos para que seja possível construir metodologias (roadmaps) ajustadas às suas necessidades (CARVALHO; RABECHINI JR., 2006, 2007).

Shenhar (2001) enfatiza também a importância da categorização de projetos e propõe uma classificação em quatro níveis de incerteza tecnológica e três níveis de complexidade dos sistemas, de acordo com a hierarquia de sistemas e subsistemas. Para o autor, as organizações 
deveriam adicionar um passo formal de classificação de projetos à fase de planejamento.

Não obstante, a literatura recente da área de gestão de projetos vem apontando para o aumento de importância das questões organizacionais em face do foco único no projeto em si (KERZNER, 2001; PMI, 2004; ENGWALL, 2003; IBERT, 2004; CARVALHO; RABECHINI Jr., 2006, 2007).

Engwall (2003) é enfático em afirmar que se devem relacionar os projetos ao seu contexto histórico e organizacional. Entender projetos em uma perspectiva de sistema aberto e em uma abordagem contingencial pode minimizar conflitos na sua execução. Portanto, alinhar as metodologias de gerenciamento e estruturas organizacionais em projetos torna-se necessário.

Carvalho e Rabechini Jr. (2005) destacam que o gerenciamento de projetos só agrega valor se tratado em âmbito organizacional, pois este consiste em um processo de mudanças culturais profundas, que envolve a construção de vários níveis de competências, a criação de uma metodologia própria de gerenciamento, com um projeto de estrutura organizacional que suporte a área de gestão de projetos e a carreira do gerente de projetos. Além disto, é necessário alinhar a atividade de projetos externamente com o ambiente competitivo e internamente com as estratégias organizacionais (CARVALHO; RABECHINI Jr., 2006, 2007).

Partindo desta perspectiva organizacional do gerenciamento de projetos traçada nesta seção, pretende-se aprofundar as questões específicas de estruturas organizacionais no ambiente de projetos.

\subsection{Estratégia e estruturas de gerenciamento de projetos}

Porter (1996) argumenta que, embora a eficiência operacional seja necessária num cenário competitivo, ela não é uma forma de estratégia e tão pouco pode substituí-la. Uma empresa pode superar o desempenho de seus rivais somente se conseguir estabelecer uma vantagem competitiva que poderá ser mantida. Portanto, a empresa dever buscar fornecer valor para o cliente, criar valor para si própria ao menor custo ou fazer as duas coisas.

A eficiência operacional pode ser definida como a maneira de executar atividades similares melhor do que seus competidores. Para atingir eficiência, foram desenvolvidas várias ferramentas gerenciais, como por exemplo: qualidade total, benchmarking, outsourcing, partnering e reengenharia. No entanto, essas ferramentas são necessárias, porém não suficientes, para substituir a estratégia de uma empresa. Por outro lado, uma posição estratégica significa executar atividades diferentes de seus concorrentes ou executar atividades similares de uma forma diferente. Com isso, um dos problemas de focar somente em eficiência operacional é o fato de poucas empresas conseguirem competir com sucesso por um extenso período, pois rapidamente os padrões de excelência são alcançados pela concorrência corroendo a vantagem competitiva (PORTER, 1996).

A eficiência operacional seduz empresas preocupadas em medir desempenho. Decidir por uma estratégia é difícil, trade-offs podem assustar e ao escolher um posicionamento assume-se um risco de uma decisão errada. Nesse cenário, a estratégia parece limitar o crescimento da empresa, pois, ao se escolher um grupo para servir, se esta abdicando das receitas dos grupos preteridos.

Uma visão estratégica não significa uma visão estática do cenário competitivo e uma empresa pode mudar sua estratégia se houver uma mudança significativa na estrutura do setor. Entretanto, a escolha de uma nova posição deve ser direcionada pela habilidade para fazer novas trocas e desenvolver um novo sistema de atividades complementares para obter vantagem sustentável.

Em síntese, a necessidade de inovação em processos, bens e serviços têm se tornado essencial para a sobrevivência das organizações. Por outro lado, as estruturas organizacionais concebidas para operações rotineiras não são eficientes em gerar as respostas necessárias em um ambiente turbulento (EISENHARDT, 1989; ENGWALL, 2003; IBERT, 2004; AUBRY et al., 2008).

Diante desta demanda do ambiente competitivo, uma revolução vem ocorrendo na introdução e desenvolvimento de novas estruturas organizacionais. Os executivos perceberam que as organizações devem ser mais dinâmicas, ou seja, elas devem ser capazes de se reestruturar rapidamente conforme as necessidades do mercado (KERZNER, 2001).

Há 50 anos, as empresas podiam sobreviver com somente uma ou talvez duas linhas de produtos. Com isso, a estrutura funcional satisfazia as necessidades de controle dos projetos. Com o passar dos anos, as empresas descobriram que sua sobrevivência dependia de uma diversificação de seus bens, isto é, possuir múltiplas linhas de produtos, e uma grande integração das tecnologias. Conforme a complexidade das organizações aumentava, os gerentes descobriam que as atividades de projeto não estavam sendo integradas de maneira efetiva (PATAH; CARVALHO, 2002).

Como alternativas à rigidez da estrutura organizacional funcional ou tradicional surgiram as estruturas projetizada e matricial. Para Galbraith (1971) existe um contínuo variando de acordo com a influência relativa do gerente de projeto e dos gerentes funcionais envolvidos. Em um extremo está a organização funcional e no outro extremo do espectro encontra-se a organização por projeto.

A estrutura matricial, uma combinação das estruturas funcional e projetizada, ainda pode ser dividida em matricial fraca, equilibrada e forte (PATAH; CARVALHO, 2002).

A estrutura funcional coloca o projeto a ser executado dentro de um dos departamentos técnicos da empresa. Com isso o responsável pelo projeto passa a ser o gerente funcional deste departamento (PATAH, 2002). 
Segundo Kerzner (2001), a maior vantagem da estrutura projetizada é que um único indivíduo, o gerente de projetos, mantém autoridade completa sobre o projeto como um todo. No entanto, este tipo de estrutura cria divisões dentro da empresa.

Segundo Meredith e Mantel (2000), a estrutura projetizada, mais nova que a estrutura funcional, vem apresentando um rápido crescimento nas últimas décadas. Os autores argumentam que muitas são as razões para a popularização deste tipo de estrutura organizacional, agrupando-as em quatro tópicos principais. Primeiramente, a velocidade de resposta e a orientação ao mercado tornaram-se uma exigência para a obtenção de sucesso nos dias de hoje. Em segundo lugar, o desenvolvimento de novos bens: produtos, processos ou serviços regularmente requerem informações das mais diferentes áreas de conhecimento. Em terceiro lugar, a rápida expansão de novidades tecnológicas em praticamente todas as áreas das empresas tende a desestabilizar-lhes a estrutura. Por fim, uma grande parte da alta administração das organizações não se sente muito confiante no entendimento e na coordenação de todas as atividades que ocorrem dentro de suas organizações.

A estrutura matricial combina a estrutura funcional e a projetizada para formar uma estrutura híbrida com características das duas "estruturas mãe". Em paralelo à estrutura funcional, sob a responsabilidade dos gerentes funcionais, são criados grupos de projeto, sob a responsabilidade de gerentes de projetos. Os grupos de projeto utilizam as mesmas pessoas que pertencem aos setores funcionais. Estes passam a ter dois tipos de trabalho, um relativo ao seu setor funcional e outro relativo ao projeto do qual estão participando; e dois "chefes" diferentes, o gerente funcional e o gerente do projeto (PATAH, 2004).

A estrutura matricial pode apresentar-se sob diversas formas. A primeira delas é a estrutura matricial fraca. Ela é mais parecida com a estrutura funcional e os gerentes funcionais possuem um maior poder em comparação aos gerentes de projeto. Outro tipo é a estrutura matricial forte. Este tipo de estrutura aproxima-se mais da estrutura projetizada, na qual os gerentes de projeto possuem uma maior influência sobre os funcionários da empresa do que os gerentes funcionais. Uma terceira estrutura matricial é a estrutura matricial equilibrada, em que os gerentes de projeto e os gerentes funcionais possuem o mesmo nível de influência sobre o trabalho e as pessoas que o executam (PATAH, 2004).

Um estudo empírico realizado por Larson e Gobeli (1999), com amostra de 546 projetos de desenvolvimento, cujo objetivo era investigar a importância da estrutura de gestão de projetos para seu sucesso, revelou que o sucesso varia de acordo com o tipo de estrutura de projeto usada, mesmo quando se consideram fatores contextuais. Para os autores, os projetos que contam com uma organização funcional apresentaram menos sucesso do que aqueles que utilizaram a matricial e a projetizada. Os autores destacam que não foram encontradas diferenças significativas entre as estruturas matriciais (balanceada e forte) e a projetizada no que concerne aos parâmetros técnicos e o resultado geral, já nos parâmetros cumprimento de prazos e controle de custos houve diferença significativa.

Não obstante, para Aubry et al. (2008) a organização orientada a projeto (project-oriented organization) deve atender às complexidades relacionadas a este novo cenário, superando a perspectiva tradicional de eficiência. Desta forma, é necessário dar espaço para estruturas dinâmicas e articuladas na organização, capazes de se adaptar às estratégias da organização e ao ambiente, este enfoque é importante, mas não substitui a gestão organizacional global (global organizational management). Estes autores sugerem que o escritório de gerenciamento de projetos (Project Management Office - PMO) é um aspecto fundamental da gestão de projetos em âmbito organizacional, pois alinha estratégias, projetos e estruturas, conforme será discutido na próxima seção deste artigo.

As configurações de estruturas de gerenciamento de projetos devem ser analisadas levando-se em consideração a estratégia genérica da corporação e a estratégia de manufatura, segundo seus critérios e dimensões competitivas, e a localização na matriz volume e variedade (PORTER, 1979; SLACK et al., 1996).

Quanto à escolha da matriz volume-variedade de Slack et al. (1996), esta se deveu pela sua importância e larga aplicação para a teoria de gestão da produção.

Segundo Carvalho et al. (2003) é possível identificar diferentes tipos de organizações num continuum de projetos a processos contínuos. Este continuиm pode ser ilustrado pela matriz volume-variedade apresentada na Figura 1. Segundo Slack et al. (1996), um projeto está no limite em que o volume é mínimo (único), a variedade é máxima (singular) e, o incremento de capacidade, integração e automação da tecnologia são mínimos. Desta forma, projetos demandam tratamento diferenciado no que concerne ao

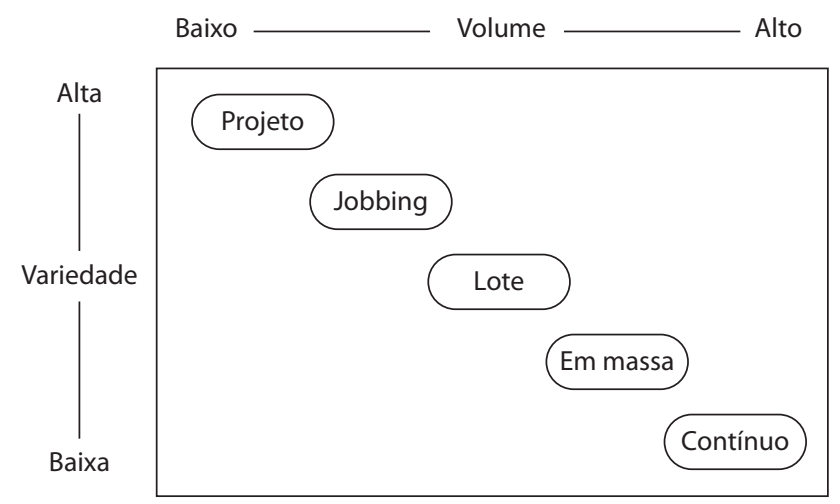

Figura 1. Matriz volume-variedade e estratégias de manufatura. (Fonte: adaptada de Slack et al., 1996.) 
seu gerenciamento, habilidades, técnicas e ferramentas específicas.

\subsection{0 project management office}

O gerenciamento de projetos está cada vez mais presente nas organizações ágeis e flexíveis. Empresas ao redor do mundo estão enviando seus funcionários para fazerem treinamento com o objetivo de melhorar o controle sobre seus projetos. Com isso os gerentes de projetos estão se tornando melhores em completar seus projetos no prazo, dentro do orçamento e de acordo com o escopo. Apesar disso, há uma emergente preocupação de que o gerenciamento de projetos deve ser controlado no nível organizacional e não individual. Reconhecendo isto, recentemente tem existido um grande esforço em direção à criação e à manutenção de um departamento chamado Project Management Office (HALLOWS, 2002).

Existem muitas estruturas de gerenciamento de projetos que são utilizadas para gerenciar projetos nos dias de hoje. Provavelmente a de maior sucesso é o PMO (Project Management Office). O conceito do PMO apareceu no final da década de 50 e começo da década de 60 (KERZNER, 2002). O PMO é uma estrutura que se preocupa com a aplicação dos conceitos de gerenciamento de projetos dentro de uma organização.

O PMO pode ser definido com uma entidade organizacional estabelecida para auxiliar os gerentes de projeto e os times da organização na implementação dos princípios, práticas, metodologias, ferramentas e técnicas do gerenciamento de projetos (DAI, 2001). Ele se apresenta como a estrutura mais robusta para a condução das atividades de um projeto (LITKE, 2002).

A maneira como os escritórios de projeto são estruturados em uma organização e o que eles fazem varia de organização para organização. Contudo, entre as funções dos escritórios de projeto, existem três principais áreas: desenvolvimento, suporte e controle. As funções de desenvolvimento são aquelas que envolvem o recrutamento, o treinamento e o desenvolvimento dos gerentes de projeto. As funções de suporte são aquelas que ajudam os gerentes de projeto a fazerem seu trabalho de uma maneira melhor através do oferecimento de assistência e clareza com os processos do gerenciamento de projetos. As funções de controle são aquelas da gerência funcional e incluem: a avaliação de gerentes de projeto, a alocação de gerentes de projeto a projetos, a garantia de que os deliverables dos projetos são produzidos e se apresentam com uma qualidade adequada e o estabelecimento de padrões. A implementação de um PMO pode ser desafiadora, mas ela não é um novo território. Muitas organizações, grandes e pequenas, estão percebendo os benefícios que um controle consistente sobre seus projetos pode oferecer (HALLOWS, 2002).

Porém, deve-se tomar cuidado para não transformarmos o PMO de uma empresa em um departamento puramente burocrático. Burghardt (2000) diz que um PMO não deve se transformar em um mero e degradante acumulador e distribuidor de papéis.

Existem muitos aspectos que deveriam ser analisados depois que a decisão de implementação do PMO foi tomada. Alguns destes aspectos são: o envolvimento da alta gerência, dos gerentes funcionais e dos gerentes de projeto da organização, o compromisso da organização com a metodologia de gerenciamento de projetos e os benefícios que a nova estrutura irá trazer à organização.

Martins et al. (2005) destacam a importância do escritório de gerenciamento de projetos respeitar a cultura da empresa, em especial no que concerne ao desenvolvimento das competências em gerenciamento de projetos.

Dai e Wells (2004), por sua vez, alertam para a existência de um conjunto de fatores motivacionais e fatores ambientais, relacionados à implantação de escritórios de gerenciamento de projetos. Os autores destacam três fatores motivacionais: melhoria do gerenciamento de projetos, existência de uma abordagem de gerenciamento de projetos comum e a melhor utilização dos recursos em um ambiente de múltiplos projetos. Já como fatores ambientais, os autores destacam o apoio da alta gerência e a ligação do PMO ao nível estratégico da organização.

Corrobora esta visão Kerzner (2003), que destaca também o apoio da alta gerência, e avança para algumas questões relacionadas aos riscos relacionados à implementação do PMO na organização. Este risco refere-se principalmente à geração de conflitos que prejudiquem a sua implementação. $\mathrm{O}$ autor conclui que o escritório de projetos pode trazer inúmeros benefícios para as organizações, no entanto a sua implantação deve seguir um planejamento estratégico para realizar estes ganhos.

Thorn (2003) demonstrou um avanço no desempenho dos projetos após a implantação do PMO, tomando como base as métricas de controle tradicionais: cronograma, orçamento e escopo. $\mathrm{O}$ autor destaca que após a implementação do PMO no caso estudado, $79 \%$ dos projetos estavam em conformidade com as estimativas de prazo, $89 \%$ em conformidade com as estimativas orçamentárias e 74\% em conformidade com a métrica de escopo. $\mathrm{O}$ autor destaca alguns fatores importantes na implementação do PMO: utilização de metodologias e ferramentas; estabelecimento e monitoramento de métricas de desempenho dos projetos; identificação de fatores críticos de sucesso; e integração dos fatores mencionados.

Johnson et al. (2002) propõem que a implantação de um PMO utilize uma abordagem orientada a processos em seis fases: escopo e arquitetura dos processos; modelagem da cadeia de processos; integração dos processos; otimização e análise dos processos; treinamento; e aperfeiçoamento contínuo.

Segundo uma revisão literária apresentada por Dai e Wells (2004), é possível identificar seis responsabilidades 
principais para um escritório de gerenciamento de projetos, quais sejam: desenvolvimento e manutenção de padrões e métodos de gerenciamento de projeto; desenvolvimento e manutenção do histórico dos projetos; suporte administrativo ao projeto; fornecimento de recursos humanos e assistência no recrutamento; consultoria e direcionamento em gerenciamento de projeto; e organização de treinamentos em gerenciamento de projeto.

A literatura apresenta diversas taxonomias de PMO segundo suas características e funções (DINSMORE, 1998; VERZUH, 1999; CASEY; PECK, 2001; HILL, 2004; DESOUZA; EVARISTO, 2006).

Uma das primeiras classificações propostas foi a de Dinsmore (1998). O autor propõe cinco modelos de PMO: Autonomous Project Team - APT; Project Support Office - PSO; Project Management Center of Excellence - PMCOE; Program Management Office - PrgMO; e Chief Project Officer - CPO.

Quando uma organização realiza alguns projetos autônomos, a função de gerenciamento de projetos permanece dentro do próprio projeto. Esta característica apresenta-se na estrutura do tipo APT. A fonte de informação sobre práticas de gerenciamento de projetos, neste caso, vem da experiência anterior e da prática dos líderes de projeto. Todos os custos da equipe do projeto são alocados nele. A organização não fornece apoio e todas as funções de gerenciamento de projetos são realizadas pela equipe do projeto. A função deste tipo de PMO é gerenciar o projeto em toda a sua integridade, com isso, a responsabilidade total pelo sucesso do projeto reside no gerente do projeto.

O PSO fornece apoio técnico e administrativo, ferramentas e serviços aos vários gerentes de projeto simultaneamente, auxiliando no planejamento, na programação e na condução das mudanças de escopo e no gerenciamento de custos dos projetos. Os recursos envolvidos são alocados nos projetos, tanto internos quanto externos, dependendo da natureza e da estrutura contratual dos projetos. Algumas vezes, pessoas do PSO são emprestadas durante a fase inicial ou mesmo ao longo de um projeto. A responsabilidade pelo sucesso do projeto não reside no PSO, mas nos gerentes de projetos que utilizam seus serviços.

O Project Management Center of Excellence é o ponto focal da experiência em projetos, mas não assume a responsabilidade por seus resultados. Ele aparece como uma despesa geral de overhead, não sendo alocada diretamente nos projetos. A tarefa do PMCOE é em grande parte de natureza missionária: disseminar a ideia, converter os incrédulos e transformar os adeptos em profissionais, sendo encarregado pelas metodologias. Ele mantém abertos os canais de informação entre os projetos e a comunidade externa ao gerenciamento de projetos.

O Program Management Office gerencia os gerentes de projetos e é, em última instância, responsável pelos resultados dos projetos. Em grandes corporações, o PrgMO concentra seus esforços nos projetos prioritários. Os demais projetos são gerenciados por departamentos ou unidades, e recebem o apoio do PrgMO conforme necessário. O PrgMO, por natureza, compreende as funções do PMCOE e, em alguns casos, as do PSO. Para que um PrgMO funcione adequadamente são necessários: poder, prioridade corporativa e controle em âmbito empresarial.

A responsabilidade do $\mathrm{CPO}$ consiste em cuidar e alimentar o portfolio de projetos da organização, desde o estágio de decisão de negócios à sua implementação final. Entre as atividades do CPO podem ser citadas as seguintes: envolvimento nas decisões de negócio que resultem em novos projetos, planejamento estratégico de negócios, estabelecimento de prioridades e negociação de recursos para projetos, supervisão da implementação de projetos estratégicos, responsabilidade pelo sistema de gerenciamento de projetos em nível empresarial, desenvolvimento da conscientização e da capacidade em gerenciamento de projetos através da organização, avaliação periódica de projetos, incluindo a decisão de descontinuar projetos, gerenciamento de stakeholders de alto nível, facilitação e mentoring.

Cabe ressaltar que, embora Dinsmore (1998) defenda os cinco modelos como evolutivos, estes podem ter maior aderência a um específico tipo de organização face ao papel do gerenciamento de projeto no tipo de estratégia corporativa.

Hill (2004) apresenta também um modelo de competências evolutivo de PMO em cinco níveis de progressão, do mais básico até o centro de excelência. Segundo o autor, é possível identificar também pelo modelo o grau de maturidade da organização, desde o controle do gerenciamento até o alinhamento estratégico com o negócio. Observa-se forte semelhança nos modelos de Dinsmore (1998) e Hill (2004).

Desouza e Evaristo (2006) também adotam uma classificação de PMO em níveis estratégicos, táticos e operacionais. A partir deste recorte, os autores propõem quatro arquétipos de PMO, baseados em duas dimensões: administrativa e intensiva em conhecimento. Enquanto a dimensão administrativa tem como foco oferecer suporte através de informações sobre os projetos e seus recursos, a intensiva em conhecimento tem foco nas melhores práticas e lições aprendidas de maturidade. Os quatro arquétipos de PMO propostos são: suporte (papel administrativo); gerente de informação (gerencia, monitora e controla as informações sobre os projetos); gerente de conhecimento (gerencia a adoção das melhores práticas; faz o mentoring e treinamento; além de ter responsabilidade pelo sucesso do projeto); e coach (é o centro de excelência da gestão de projetos na organização).

Casey e Peck (2001) apresentam três tipos de PMO: estação meteorológica, torre de controle e esquadrão de comando. O primeiro tipo, estação meteorológica, 
apenas reporta o andamento dos projetos, mas não tenta influenciá-los. Já o PMO do tipo torre de controle é sugerido quando a empresa apresenta problemas de treinamento de pessoal, metodologias caras e pouco utilizadas ou com trocas frequentes de métodos e ferramentas; altos executivos com pouca compreensão ou visão equivocada sobre gerenciamento de projetos. O terceiro tipo, em geral encontrado em organizações cujo negócio principal é desenvolver projetos, é o esquadrão de comando, com foco em multiprojetos.

Finalmente, apresenta-se a tipologia de PMO proposta por Verzuh (1999), que não advoga o caráter evolutivo dos escritórios, quais sejam: centro de excelência (Center of de Excellence-CE); escritório de suporte aos projetos (Project Support Office - PSO); escritório de gerenciamento de projetos (Project Management Office - PMO); escritório de gerenciamento de programas (Program Management Office - PrgMO); e o escritório de controladoria de projetos (Accountable Project Office - ACO). Que apresentam características semelhantes às propostas por Dinsmore (1998) e Hill (2004).

\section{Aspectos metodológicos da pesquisa}

Nesta seção são apresentados o framework teóricoconceitual de análise das estruturas e estratégias de manufatura e os principais aspectos metodológicos da pesquisa de campo.

\subsection{Estrutura teórica-conceitual de análise: estruturas e estratégias}

Nesta seção buscou-se delinear um framework para alinhar os modelos de estruturas organizacionais de projetos com as estratégias de manufatura, uma vez que não foi possível encontrar modelo equivalente na literatura nos campos da gestão de projetos e da gestão de operações.

A primeira análise a ser feita relaciona-se à importância da metodologia de gerenciamento de projetos para empresas. Esta análise pode ser feita através da proporção de projetos no volume de vendas brutas da empresa. Quanto mais alta a proporção, maior a importância da metodologia de gerenciamento de projetos e a necessidade da presença de um PMO. Quando a proporção de projetos é baixa, a metodologia de gerenciamento de projetos pode ser implementada através de uma estrutura funcional, matricial ou projetizada.

O segundo passo consiste em, baseando-se na análise simultânea da matriz volume-variedade, com as diferentes estruturas organizacionais, relacioná-las com as estratégias de manufatura, quando se leva em conta uma empresa com baixa proporção de projetos. A Figura 2 apresenta os diferentes tipos de estrutura para projetos distribuídos em uma matriz volume-variedade. Conforme mencionado, estas estruturas aplicam-se a organizações que gerenciam projetos sem possuírem um PMO estabelecido.

A estrutura funcional é mais bem aplicada a projetos executados dentro dos departamentos técnicos da empresa. $\mathrm{Na}$ estrutura projetizada o gerente de projetos mantém uma autoridade completa sobre o projeto como um todo. A estrutura matricial cria grupos de projeto, sob a responsabilidade dos gerentes de projeto e utiliza as mesmas pessoas que pertencem aos setores funcionais. Estas estruturas são aplicadas a organizações que gerenciam projetos, porém não sentem a necessidade da presença de um departamento específico para o gerenciamento dos projetos, o PMO.

Quando a proporção de projetos é alta em uma empresa, a presença de um PMO torna-se extremamente necessária. Então, baseando-se na análise simultânea da matriz volume-variedade com os tipos de PMOs existentes, pode-se relacioná-los com cada uma das estratégias de manufatura.

O APT trabalha bem quando o projeto não tem nenhuma grande necessidade de manter uma ampla interface com o restante da organização e a empresa tem pouca experiência em projetos. Nesse caso este tipo de estrutura seria mais bem aplicado a uma empresa com processo de produção contínuo que, eventualmente, irá implantar um projeto.

Os PSOs são especialmente aplicáveis em cenários em que: os projetos sejam liderados por gerentes de projetos fortes; exista dentro da organização a conscientização para o gerenciamento de projetos; haja uma forte necessidade de documentação de apoio e de rastreamento formalizado; e existam muitos projetos acontecendo. Os PSOs seriam mais bem aplicados aos projetos de organizações com estratégia de manufatura localizada entre a produção em massa e a produção por lotes, com uma grande variedade de projetos sendo implementados.

A abordagem PMCOE é particularmente adequada para corporações com responsabilidades globais, com projetos de diferentes naturezas (como sistemas de informação, marketing, engenharia e mudança organizacional), e que

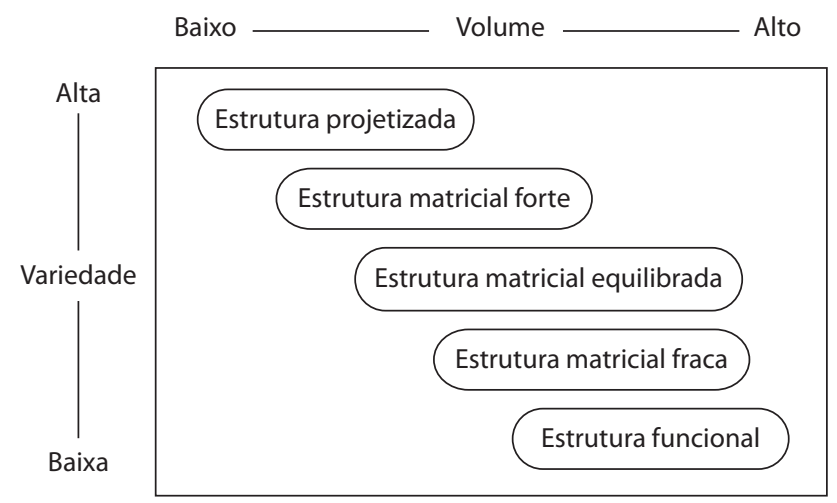

Figura 2. Estruturas de gerenciamento de projetos localizadas na matriz volume-variedade. (Fonte: elaborada pelos autores.) 
prefiram uma abordagem soft para influenciar a cultura interna. Este tipo de estrutura de PMO seria mais bem aplicado em organizações com estratégia de manufatura localizada entre a produção em lotes e a produção por jobbing, também com uma grande variedade de projetos.

Os PrgMOs são normalmente aplicáveis quando o gerenciamento corporativo tenha se comprometido a gerenciar empreendimentos prioritários por projeto, quando há maturidade organizacional adequada para que uma superintendência de projeto funcione eficazmente, e quando não gerenciar por projetos signifique fortes consequências negativas. O PrgMO deveria ser aplicado somente em empresas com estratégias de manufatura voltadas exclusivamente para projetos, ou localizadas entre as estratégias de manufatura de jobbing e projetos. Estes tipos de estratégia de manufatura permitem que todas as vantagens do PrgMO sejam obtidas.

O CPO é aplicável em organizações globais, orientadas para empreendimentos, multissetoriais e que exijam os resultados de projetos múltiplos e complexos no prazo previsto. O CPO, por sua vez, também só deveria ser aplicado em grandes empresas com estratégias de manufatura totalmente voltadas para projetos.

A Figura 3 apresenta os diferentes tipos de PMOs distribuídos em uma matriz volume-variedade. Cinco modelos de PMO podem ser observados nesta Figura: Autonomous Project Team (APT); Project Support Office (PSO); Project Management Center of Excellence (PMCOE); Program Management Office (PrgMO); e Chief Project Officer (CPO).

Com base nas informações coletadas junto às empresas analisadas nos casos, é possível posicionar estas empresas em uma das estratégias de manufatura da matriz volumevariedade, apresentadas na Figura 1.

Em um segundo momento, tendo como base a estratégia de manufatura de cada uma das empresas dos casos, fez-se o alinhamento destas com as estruturas de

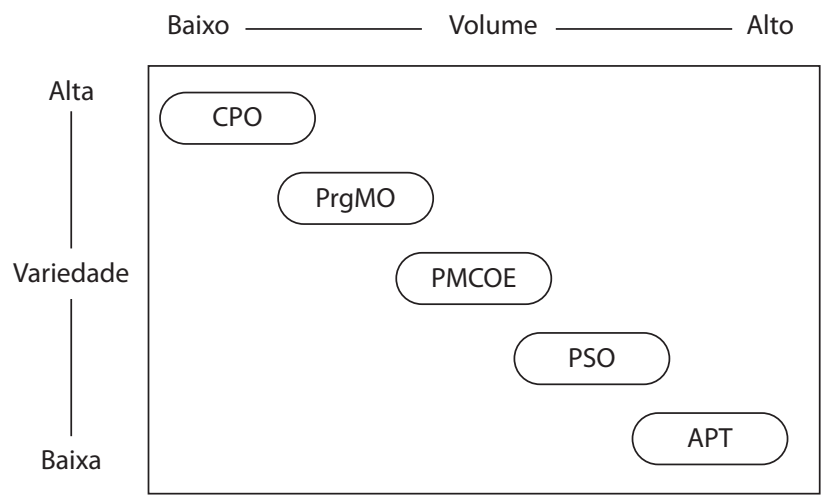

Figura 3. Tipos de PMOs localizados na matriz volume-variedade. (Fonte: elaborada pelos autores.) gerenciamento de projetos, através do modelo apresentado nas Figuras 2 e 3.

\subsection{Metodologia de pesquisa}

A opção metodológica adotada foi de estudo de múltiplos casos, selecionando três estudos de caso de empresas dos segmentos de cosméticos, eletro-eletrônico e aeroespacial. Os principais elementos investigados no estudo de campo foram: caracterização da empresa, o papel estratégico da atividade de projeto, a estrutura de gerência de projetos existente, os investimentos em atividades relacionadas ao gerenciamento de projetos e a caracterização das competências das equipes de projeto.

Foi utilizado como critério para seleção dos estudos de caso o modelo da matriz volume-variedade, apresentado na Figura 1, buscando-se estudar organizações em diferentes regiões da referida matriz. Portanto, cada um destes casos serve um propósito específico dentro do escopo global da investigação. A empresa do segmento de cosméticos localiza-se no extremo inferior direito da matriz. Já a empresa do segmento eletro-eletrônico localiza-se no meio da matriz e a empresa do segmento aeroespacial localiza-se no extremo superior esquerdo da mesma matriz, conforme a Figura 4. Desse modo, os três casos se complementam e fornecem uma análise mais robusta para o alinhamento desejado neste trabalho. No que se refere a esta escolha vale a pena ressaltar que os casos foram analisados individualmente, mas que se agregam no sentido de dar uma contribuição mais rica ao entendimento do fenômeno a ser estudado. Desse modo os casos se complementaram, produzindo resultados contrastantes por razões previsíveis, buscando-se com isso obter replicação teórica. Entendeu-se que, por cobrirem as três principais áreas distintas da matriz já citada, a quantidade de casos atenderia ao objetivo da pesquisa.

Por fim cabe ressaltar as fontes de evidências utilizadas para o levantamento de informações nos casos. Foram utilizadas, com diferentes intensidades, as seguintes formas: documentação, registro de arquivo, entrevistas e a observação direta.

As proposições que embasaram o trabalho foram:

- A escolha da estrutura do gerenciamento de projetos, suas características e seus processos de implantação dependem do tipo de organização e do papel da atividade de projetos enquanto fonte de vantagem competitiva.

- As organizações buscam o alinhamento do tipo adequado de estrutura de gerenciamento de projetos à sua estratégia de manufatura, com o objetivo de maximizar os benefícios proporcionados por ele.

Com o protocolo, buscou-se refletir as proposições, em função da questão da pesquisa, incluindo os instrumentos de levantamento de dados que foram praticados nos respectivos 
estudos. Nas empresas analisadas foram feitas entrevistas, contemplando os seguintes aspectos:

- estratégias empresariais, enfocando o processo de implementação das estratégias elaboradas pelas empresas;

- gerenciamento de projetos, mais especificamente a estrutura de gerenciamento de projetos e seus principais papéis e funções.

O roteiro das entrevistas foi dividido, basicamente, em três seções. A primeira seção visava obter informações gerais das empresas incluindo o porte de seus projetos, avaliado de acordo com o número de pessoas que participam das equipes, do valor dos projetos em reais e de sua duração. A segunda seção buscou compreender as estratégias empresariais das organizações, levando em conta os produtos produzidos por elas, sob duas óticas: volume de produtos produzidos e variedade. Já a terceira seção preocupou-se com as questões referentes ao gerenciamento de projetos das organizações, mais especificamente com os tipos de estruturas organizacionais montadas para a coordenação dos projetos, seus papéis e suas funções nas organizações.

Estas informações foram utilizadas na busca do estabelecimento de uma relação entre a estrutura de gerenciamento de projetos das organizações analisadas com suas estratégias de manufatura.

Para a coleta de informações, foram feitas no mínimo 5 entrevistas com gerentes de projetos, responsáveis por áreas de gerenciamento de projetos e gerentes funcionais de cada uma das organizações, contemplando os seguintes aspectos:

- estratégias empresariais e estratégias de manufatura, enfocando o processo de implementação das estratégias elaboradas por estas empresas;

- gerenciamento de projetos, mais especificamente a estrutura organizacional de projetos e seus principais papéis e funções.

Após a realização das entrevistas, foram feitas comparações das funções das estruturas adotadas na prática pelas empresas estudadas, com as funções que, idealmente, seriam as mais adequadas para as empresas de cada segmento de negócio, quando da implementação de suas estratégias de manufatura através de uma estrutura deste tipo.

\section{Análise de resultados}

Nesta seção faz-se a análise dos resultados obtidos nas três empresas estudadas, pertencentes aos setores de cosméticos, eletro-eletrônico e aeroespacial; relacionando-se as estruturas com as estratégias de manufatura.

A empresa analisada do setor de cosméticos é uma empresa brasileira, com mais de 30 anos de existência e possui mais de 2 mil colaboradores. A empresa fabrica cosméticos e produtos de higiene e de perfumaria. Com produção de mais de 100 milhões de itens por ano, que compõe um portfolio de 450 produtos, a empresa responde por $14 \%$ do mercado em que opera. Ela distribui seus próprios produtos e os coloca no consumidor final por meio de venda direta, atingindo 5 mil municípios brasileiros. A empresa possui fábrica e sede central na cidade de São Paulo - SP.

A empresa do segmento eletro-eletrônico possui mais de 8 mil funcionários e está presente no País a mais de 100 anos. Suas diversas divisões atuam nos segmentos de telecomunicações, indústria, energia e medicina. A empresa possui fábricas em diversas regiões do País, tais como em Curitiba-PR e Manaus-AM. Sua sede está localizada na cidade de São Paulo-SP e ela possui filiais e escritórios de vendas nas principais cidades brasileiras. Segundo cálculos da matriz, estima-se que, para toda a empresa em nível mundial, $60 \%$ das vendas são gerenciadas como projetos. Os projetos nesta organização variam de $\mathrm{R} \$ 10 \mathrm{mil}$ a R \$ 20 milhões em custo e de alguns dias a alguns anos em prazo de execução.

Com mais de 30 anos de experiência em projeto, fabricação, comercialização e pós-venda a empresa do segmento aeroespacial alcançou uma alta posição graças à excelência de seus produtos e à tecnologia de ponta empregada no segmento aeronáutico. A empresa tem uma base global de clientes e importantes parceiros de renome mundial, o que resulta em uma significativa participação no mercado. Atualmente emprega mais de 12 mil funcionários, contribuindo, adicionalmente, para a geração de mais de 3 mil empregos indiretos. Atua nos mercados de aviação comercial, aviação de defesa, nos quais desempenha um papel estratégico no sistema de defesa brasileiro, tendo fornecido mais de $50 \%$ da frota da força aérea brasileira e aviação corporativa, e ainda pretende conquistar parcela significativa do mercado de jatos executivos de médio porte. A empresa está sediada em São José dos Campos-SP onde também possui fábrica. Possui também unidades em Botucatu-SP e Gavião Peixoto-SP. Além do Brasil, a empresa está presente nos seguintes países: EUA, Austrália, França, China e Cingapura.

\subsection{Relacionamento entre os modelos de estruturas e as estratégias das empresas analisadas}

Em duas das três empresas analisadas, o gerenciamento de projetos apresenta-se como uma ferramenta de suma importância. Para estas empresas, que são dos segmentos eletro-eletrônico e aeroespacial, um PMO é o elemento estratégico utilizado para gerenciar seus projetos.

A empresa aeroespacial, que produz aviões de tamanho médio, depende muito de projetos. Mais de $95 \%$ de suas vendas brutas provêm de projetos, portanto, uma metodologia de gerenciamento de projetos é extremamente importante. 
Para a empresa do segmento eletro-eletrônico, $60 \%$ das vendas brutas provêm de projetos. Produz e instala uma grande variedade de equipamentos eletrônicos, que são, na sua grande maioria, entregues aos clientes por meio de projetos específicos adaptados às necessidades de cada um. A taxa de inovação dos produtos é extremamente alta e os produtos atualmente vendidos foram desenvolvidos no máximo três anos atrás. Basicamente, a empresa vende soluções customizadas para seus clientes.

A outra empresa, do segmento de cosméticos, pode gerenciar seus projetos com uma estrutura simples. O maior volume de vendas da empresa, mais de $90 \%$, provém da produção em massa.

Com base nas entrevistas realizadas foi possível classificar as três empresas estudadas segundo o seu volume e a sua variedade. De acordo com a Figura 4, é possível verificar a posição delas entre as estratégias de manufatura.

De acordo com os relacionamentos entre as estratégias de manufatura e os modelos de estruturas de gerenciamento de projetos e PMOs, desenvolvidos no item 3.1, as empresas dos casos analisados deveriam apresentar estruturas, teoricamente, conforme apresentado na Tabela 1.

\subsection{Classificação das estruturas de gerenciamento de projetos das empresas analisadas}

Baseando-se na análise das três empresas estudadas, é possível concluir que a empresa do segmento de cosméticos

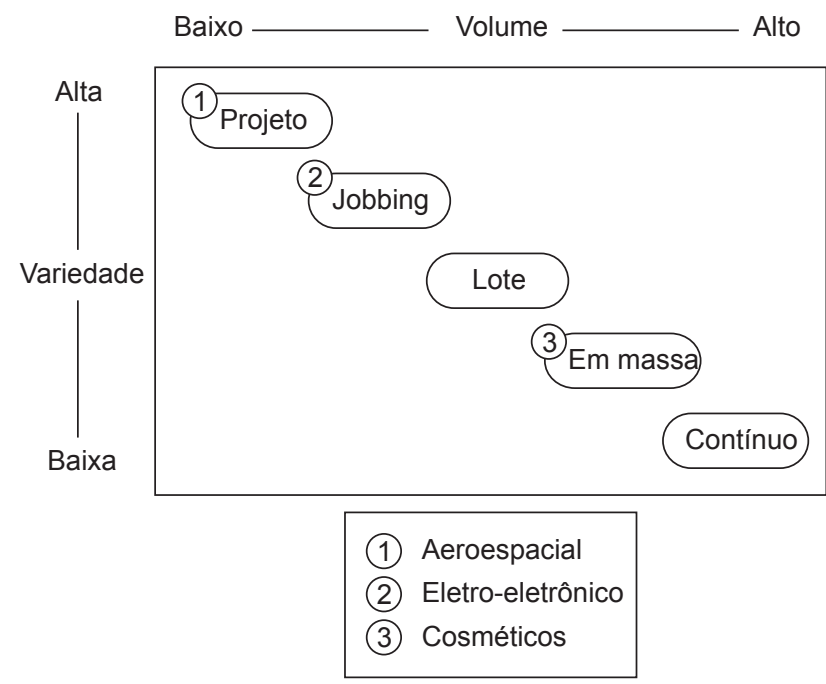

Figura 4. Distribuição das empresas na matriz volume-variedade. (Fonte: elaborada pelos autores.)

Tabela 1. Estruturas teóricas para as empresas analisadas.

\begin{tabular}{lc}
\hline \multicolumn{1}{c}{ Empresa } & Estrutura \\
\hline Cosméticos & Estrutura - Matricial Fraca \\
Eletro-eletrônico & PMO - PrgMO \\
Aeroespacial & PMO - CPO \\
\hline
\end{tabular}

(Fonte: elaborada pelos autores.) conduz seus projetos utilizando uma estrutura matricial fraca, quando necessário, conforme apresentado pelos colaboradores entrevistados nesta empresa e verificado pessoalmente durante visita à organização. Já a empresa do segmento eletro-eletrônico possui um PMO caracterizado como Project Management Center of Excellence (PMCOE). Para esta empresa o PMO é o ponto focal para a experiência em projetos, mas não assume a responsabilidade pelos resultados dos projetos. É possível verificar que os aspectos que caracterizam o PMO da empresa analisada são, na prática, os mesmos mencionados por Dinsmore (1998) e citados no item 2.2, para PMOs do tipo Project Management Center of Excellence (PMCOE), com especial atenção à localização dos gerentes de projeto fora do $\mathrm{PMO}$ e à não responsabilidade pelos resultados dos projetos. Com base nas figuras 3 e 4 e nos relacionamentos entre estratégias de manufatura e tipos do PMO desenvolvidos no item 3.1, pode-se verificar que ainda existe um espaço para a migração do PMO da empresa, atualmente do tipo PMCOE, para um PrgMO. Isto deve vir a acontecer com o decorrer do tempo, uma vez que o PMO analisado possui apenas três anos de funcionamento e, nesta primeira etapa, vem se ocupando com os aspectos relacionados à implementação dos conceitos de gerenciamento de projetos dentro desta organização. Finalmente, a empresa aeroespacial possui um PMO do modelo PrgMO e até mesmo um CPO, de acordo com a classificação de Dinsmore (1998) apresentada no item 2.2.

\section{Conclusão}

Primeiramente, através da análise das empresas estudadas, buscou-se obter a atual estrutura de gerenciamento de projetos destas organizações. Além disso, também se buscaram informações que permitissem posicionar estas empresas em uma das estratégias de manufatura da matriz volume-variedade, apresentadas na Figura 1.

Em um segundo momento, tendo como base a estratégia de manufatura de cada uma das empresas dos casos, fez-se o alinhamento destas com as estruturas de gerenciamento de projetos, apresentadas na seção 2 .

Para isto, os autores utilizaram-se do framework delineado na seção 3.1 e apresentado nas Figuras 2 e 3, que compôs a estrutura teórica-conceitual de análise proposta que buscou alinhar os modelos de estruturas organizacionais de projetos com as estratégias de manufatura.

Buscou-se com isso, não a validação do modelo, mas sugestões de que há potencial de validade. Isto porque o estudo apresentado possui duas limitações: o fato de tratar de somente três estudos de casos e o fato de a análise ocorrer somente através da matriz volume-variedade.

Através da análise das atividades das estruturas de gerenciamento de projetos dos casos estudados, é possível confirmar que os modelos e os objetivos destas estruturas 
são, em sua grande parte, os mesmos apresentados pela teoria. Portanto, de acordo com as informações obtidas das empresas analisadas e com a conceituação teórica, pode-se concluir que as estruturas das empresas estão amparadas pela teoria apresentada.

Uma estrutura organizacional de projetos é uma estrutura que cuida da aplicação dos conceitos de gerenciamento de projetos dentro de uma organização. Ela pode se apresentar sob diferentes formas: desde um grupo isolado que auxilia um projeto específico até um importante departamento no qual todos os projetos são gerenciados. Muitas organizações, grandes e pequenas estão percebendo os benefícios gerados pelo monitoramento constante de seus projetos.

Além disso, existem muitos outros aspectos que deveriam ser analisados antes que uma decisão em direção à implementação de uma estrutura organizacional de projetos seja tomada. Alguns destes aspectos são: grande envolvimento da alta gerência, dos gerentes funcionais e dos gerentes de projetos da organização, com a metodologia de gerenciamento de projetos e com os benefícios que a nova estrutura trará para a empresa. A escolha da adequada estrutura organizacional de projetos deve estar conectada com a estratégia da empresa. Este é um fator crítico de sucesso de uma implementação bem sucedida.

Como recomendações para futuras pesquisas, sugerem-se a extensão da análise a mais empresas, uma vez que a pesquisa ocorreu apenas com empresas de grande porte e de produção de bens, e a possibilidade de se fazer a aplicação conjugada de métodos adicionais de análise de estratégia nos casos trabalhados.

\title{
Alignment between project management organizational structure and manufacture strategy: a comparative analysis of multiple cases
}

\begin{abstract}
The main goal of this paper is to establish a relation between the project management organization for a company and its manufacture strategy. Another objective is also to verify whether there is an alignment between the configuration of the organizational structure and the model of the manufacture strategy for a company. The methodological approach used was the multiple cases analysis conducted in three companies of the cosmetics, electronic, and airspace sectors. The main results point to more complex project management structures in the process along the continuum between volume and variety.
\end{abstract}

Keywords: Project management. Manufacture strategy. Organizational structure. Project management office.

\section{Referências Bibliográficas}

AUBRY, M.; HOBBS, B.; THUILLIER, D. A new framework for understanding organizational project management through the PMO. International Journal of Project Management, v. 25, n. 1, p. 38-43, 2008.

BURGHARDT, M. Projektmanagement: leitfaden für die Planung, Überwachung und Steuerung von Entwicklungsprojekten. Berlin; München: Siemens Aktiengesellschaft, 2000.

CARDINAL, J. S.; MARLE, F. Project: The just necessary structure to reach your goals. International Journal of Project Management, v. 24, n. 3, p. 226-233. 2006.

CARVALHO, M. M.; LAURINDO, F. J. B.; PESSÔA, M. S. P. Information technology project management to achieve efficiency in Brazilian companies. In: KAMEL, S. (Org.). Managing globally with information technology. Hershey: [s.n.], 2003. p. 260-271.

CARVALHO, M. M.; RABECHINI Jr., R. Cadeia de valor em projetos. Mundo PM, v. 14, abril-maio, p. 48-60, 2007.

CARVALHO, M. M.; RABECHINI Jr., R. Gestão de projetos na prática: casos Brasileiros. São Paulo: Editora Atlas, 2006.
CARVALHO, M. M.; RABECHINI Jr., R. Construindo competências para gerenciar projetos: teoria \& casos. São Paulo: Editora Atlas, 2005.

CASEY, W.; PECK, W. Choosing the right PMO setup. PM Network, p. 40-47, 2001.

CLELAND, D. I.; IRELAND, L. R. Project manager's portable handbook. New York: McGraw-Hill, 2000.

DAÍ, C.; WELLS, W. An exploration of project management office features and their relationship to project performance. International Journal of Information Management, v. 22, n. 7, p. 523-532, 2004

DAI, X. C. The role of the project management office in achieving project success. 2001. Tese (Doutorado) - The George Washington University, Washington DC.

DESOUZA, K.; EVARISTO, J. Project management offices: a case of knowledge-based archetypes. International Journal of Information Management, v. 26, n. 5, p. 414-423, 2006.

DINSMORE, P. C. Winning business with enterprise project management. New York: AMACOM, 1998. 
EISENHARDT, K. Building theories from case study research. Academy of Management Review, v. 14, n. 14, p. 532-550, 1989.

ENGWALL, M. No project is an island: linking projects to history and context. Research Policy, v. 32, n. 5, p. 789-808, 2003.

HALLOWS, J. E. The project management office toolkit. New York: AMACOM, 2002.

HILL, G. Evolving the project management office: a competency continuum. Information Systems Management, v. 21, n. 4, p. 45-51, 2004.

IBERT, O. Projects and firms as discordant complements: organizational learning in the Munich software ecology. Research Policy, v. 33, n. 10, p. 1529-1546, 2004

JOHNSON, M.; JOYNER, T.; MARTIN Jr., R. Process-driven project management office implementation. AACE International Transactions, p. PM11.1-PM11.7, 2002.

KERZNER, H. Strategic planning for a project office. Project Management Journal, v. 34, n. 2, p. 13-25, 2003.

KERZNER, H. Gestão de projetos: as melhores práticas. Porto Alegre: Bookman, 2002.

KERZNER, H. Project management: a systems approach to planning, scheduling, and controlling. New York: John Wiley \& Sons, 2001

KING, W. R. The role of projects in the implementation of business strategy. In: CLELAND, D. I.; KING, W. R. (Eds.). Project management handbook. New York: Van Nostrand Reinhold, 1993.

LARSON, E. W.; GOBELI, D. H. Significance of project management structure on development success. IEEE Transactions on Engineering Management, v. 36, n. 2, p. 119-125, 1999.

LITKE, H. D. Projekt-management. München: Gräfe und Unzer, 2002.

LITKE, H. D. Projekt-management: methoden, techniken, verhaltensweisen. München; Wien: Carl Hansen, 1995.

MARTINS, A. et al. Implantação e consolidação de escritório de gerenciamento de projetos: um estudo de caso. Revista Produção, v. 15, n. 3, p. 404-415, 2005.

MEREDITH, J. R.; MANTEL Jr., S. J. Project management a managerial approach. New York: John Wiley \& Sons, Inc., 2000.
MINTZBERG, H.; HEYDEN, L. V. Organigraphs: drawing how companies really work. Harvard Business Review, p. 87-94, 1999.

MINTZBERG, H.; WATERS, J. A. Of strategies, deliberate and emergent. Strategic Management Journal, v. 6, n. 3, p. 257-273, 1985.

PATAH, L.; CARVALHO, M. M. Estruturas de gerenciamento de projetos e competências em equipes de projetos. In: ENCONTRO NACIONAL DE ENGENHARIA DE PRODUÇÃO, 22., 2002, Curitiba. Proceedings... Porto Alegre: ABEPRO, 2002. p. 1-8.

PATAH, L. Alinhamento estratégico de estrutura organizacional de projetos: uma análise de múltiplos casos. 2004. 169 f. Dissertação (Mestrado) - Universidade de São Paulo, São Paulo.

PATAH, L. Estrutura de gerenciamento de projetos em empresas. In: SIMPÓSIO DE ADMINISTRAÇÃO DA PRODUÇÃO, LOGÍSTICA E OPERAÇÕES INTERNACIONAIS, 5., 2002, São Paulo. Proceedings... São Paulo: Editora Quality Digital, 2002. p. 38-44.

PLATTS, K.; MILLS, J. Creating manufacturing strategy. In: INTERNATIONAL CONFERENCE ON INDUSTRIAL ENGINEERING AND OPERATIONS MANAGEMENT, 8., 2002, Proceedings... Porto Alegre: Technical and Organizational Integration of Supply Chains, 2002.

PROJECT MANAGEMENT INSTITUTE - PMI. A guide to the project management body of knowledge (PMBoK). Maryland, 2004.

PORTER, M. How competitive forces shape strategy. Boston: Harvard Business Review, 1979.

PORTER, M. What is strategy? Boston: Harvard Business Review, 1996.

SHENHAR, A. J. One size does not fit all projects: exploring classical contingency domains. Management Science, v. 47, n. 3, p. 394-414, 2001

SLACK, N. et. al. Administração da produção. São Paulo: Atlas, 1996.

THORN, M. B. Over troubled water: implementation of a program management office. SAM Advanced Management Journal, p. 48-59, 2003.

VERZUH, E. The fast forward MBA in project management. New York: John Wiley \& Sons, 1999.

\section{Sobre os autores}

\section{Leandro Alves Patah}

Escola Politécnica da Universidade de São Paulo

Av. Prof. Almeida Prado, trav. 2, n 128, Butantã - USP, CEP 05508-900, São Paulo - SP

e-mail: leandro.patah@uol.com.br

\section{Marly Monteiro de Carvalho}

Escola Politécnica da Universidade de São Paulo

Av. Prof. Almeida Prado, trav. 2, n 128, Butantã - USP, CEP 05508-900, São Paulo - SP

e-mail: marly.carvalho@poli.usp.br 